\title{
Interfacial Structure and Electrical Properties of Transparent Conducting ZnO Thin Films on Polymer Substrates
}

\author{
Young Soo Lim, ${ }^{1}$ Dae Wook Kim, ${ }^{1,2}$ Jong-Ho Kang, ${ }^{1,2}$ Seul Gi Seo, ${ }^{1,2}$ Bo Bae Kim, ${ }^{1,2}$ \\ Hyoung-Seuk Choi, ${ }^{1}$ Won-Seon Seo, ${ }^{1, \star}$ Yong Soo Cho, ${ }^{2}$ and Hyung-Ho Park ${ }^{2}$ \\ ${ }^{1}$ Energy and Environmental Division, Korea Institute of Ceramic Engineering and Technology, 233-5 Gasan-dong, \\ Geumcheon-gu, Seoul 153-801, Korea \\ ${ }^{2}$ Department of Materials Science and Engineering, Yonsei University, 134 Sinchon-dong, Seodaemun-gu, \\ Seoul 120-749, Korea
}

\begin{abstract}
The effects of polymer substrates on the interfacial structure and the thermal stability of Ga-doped $\mathrm{ZnO}$ (GZO) thin films were investigated. The GZO thin films were deposited on polyethylene terephthalate (PET) and polyethylene naphthalate (PEN) substrates by rf-magnetron sputtering at room temperature, and thermal stability tests of the GZO thin films on the polymer substrates were performed at $150^{\circ} \mathrm{C}$ up to $8 \mathrm{~h}$ in air. Electrical and structural characterizations of the GZO thin films on the PET and the PEN substrates were carried out, and the origins of the stable interfacial structure and the improved thermal stability of the GZO thin film on the PEN substrate were discussed.
\end{abstract}

Key words: zinc oxide, transparent conducting oxide, sputtering, thermal stability, oligomer

\section{INTRODUCTION}

Transparent conducting oxide (TCO) thin film has been extensively investigated for its numerous applications, such as displays, solar cells, and light-emitting diodes (Minami, 2000). Indium tin oxide (ITO) has dominated the TCO markets because of its high transmittance, low electric resistivity, and good stability. Nevertheless, it has been expected that rapidly increasing TCO-related markets can lead to a risk of indium shortage (Minami, 2000). Therefore, there have been strong needs to find alternative TCO materials. In this respect, $\mathrm{ZnO}$ doped with trivalent elements (e.g., $\mathrm{B}, \mathrm{Al}$, $\mathrm{Ga}$, and $\mathrm{In}$ ) is a strong candidate to replace the ITO owing to its several advantages such as low cost, material abundance, and nontoxicity (Minami, 2000; Ellmer \& Mientus, 2008).

Recently, the growth of the impurity-doped $\mathrm{ZnO}$ thin film on plastic substrate has attracted much attention for applications in flexible electronics (Kim et al., 2010). However, there have been some demerits regarding plastic substrates, such as weak interface between inorganic thin film and organic substrate, low thermal stability related to its high coefficient of thermal expansion (CTE), and easy permeation of moisture and oxygen molecules (MacDonald et al., 2005; Cheng \& Wagner, 2009). Among these problems, there have been only few reports on the interfacial structure and the thermal stability of the impurity-doped $\mathrm{ZnO}$ thin film on the plastic substrate (Kim et al., 2010, 2011), whereas the barrier coating against the moisture and gas permeation has been extensively investigated by many research groups (Fortunato et al., 2002; Koidis et al., 2009).

(C) MICROSCOPY SOCIETY OF AMERICA 2013

${ }^{\star}$ Corresponding author. E-mail: wsseo@kicet.re.kr
In this work, we report the interfacial structure and the thermal stability of Ga-doped $\mathrm{ZnO}$ (GZO) thin films grown on polymer substrates. For a comparative study, polyethylene terephthalate (PET) and polyethylene naphthalate (PEN) substrates were used for the deposition of the GZO thin films. Sputtering deposition of the GZO thin films was performed on the PET and PEN substrate at room temperature, and thermal stability tests of the films were performed at $150^{\circ} \mathrm{C}$ for up to $8 \mathrm{~h}$ in air. Structural and electrical characterizations of the GZO thin films were carried out, and the effects of the polymer substrates on the interfacial structure and the thermal stability of the GZO thin films were discussed.

\section{Materials and Methods}

Commercial PET (KDL86W-188 um, Teijin-DuPont) and PEN (Q65FA-200 um, Teijin-DuPont) films were used for plastic substrates, and GZO thin films were deposited on the substrates by rf-magnetron sputtering using a $5.5 \mathrm{wt} \%$ $\mathrm{Ga}_{2} \mathrm{O}_{3}$-doped $\mathrm{ZnO}$ target at room temperature. The base pressure was $3.0 \times 10^{-6}$ torr and the working pressure was 1 mtorr. For the sputtering gas, Ar flow of $30 \mathrm{sccm}$ was introduced into the sputtering chamber. The rf power and the sputtering time were $115 \mathrm{~W}$ and $17 \mathrm{~min}$, respectively. After the deposition, thermal stability test of the GZO thin film was carried out at $150^{\circ} \mathrm{C}$ up to $8 \mathrm{~h}$ in a convection oven.

The thicknesses of the GZO thin films grown on the PET and PEN substrates were around $140 \mathrm{~nm}$. The interfacial structure between the GZO thin film and the plastic substrate was investigated by using a transmission electron microscope (TEM, JEM-4010, JEOL). The TEM samples 


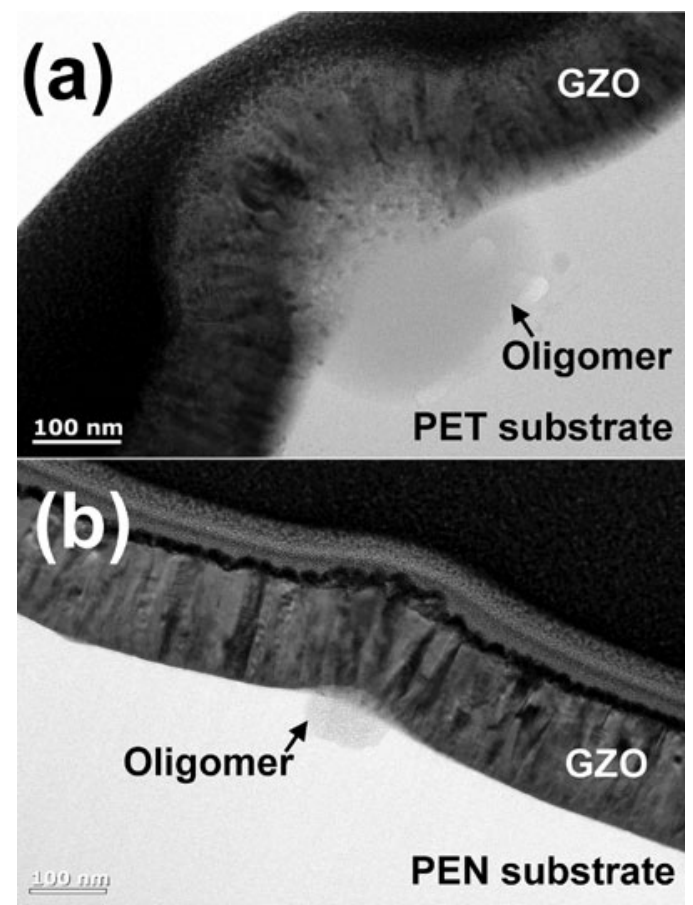

Figure 1. Cross-sectional TEM micrographs of the GZO thin films grown on (a) PET substrate and (b) PEN substrate. TEM, transmission electron microscope; GZO, Ga-doped ZnO; PET, polyethylene terephthalate; PEN, polyethylene naphthalate.

were prepared by using a focused ion beam (Helios Nanolab 600 , FEI). The behavior of the crack formation in the GZO thin film after the thermal stability tests was analyzed by using an optical microscope (Me600L, Nikon-Eclipse). The changes in the electrical properties of GZO thin films were examined by Hall measurements (HMS-3000, Ecopia).

\section{Results And Discussion}

Figures $1 \mathrm{a}$ and $1 \mathrm{~b}$ are cross-sectional TEM micrographs of as-deposited GZO thin films on the PET and the PEN substrates, respectively. Because both the PET and the PEN substrates are semi-crystalline polymers, which are composed of crystalline and amorphous phases, the locally crystallized phase of the amorphous polymer chains, i.e., oligomer, can be produced by the energetic bombardment of sputtered species during the sputtering deposition of the GZO thin films (Bishop, 2010). The oligomer phase in the PET substrate was much bigger than that in the PEN substrate. The different shapes of the oligomers depending on the type of the substrate reveal the characters of the polymer substrates. The polymer chain with a single benzene ring in the PET substrate might be more mobile than that with two fused benzene rings in the PEN substrate (Yasuda, 1978; Reichlmaier et al., 1995; Bishop, 2010). Therefore, the crystallization of the amorphous chains can be induced with a relatively large size in the PET substrate than in the PEN substrate.

The GZO thin films showed the radial distribution of the column structure in accordance with the curvature of

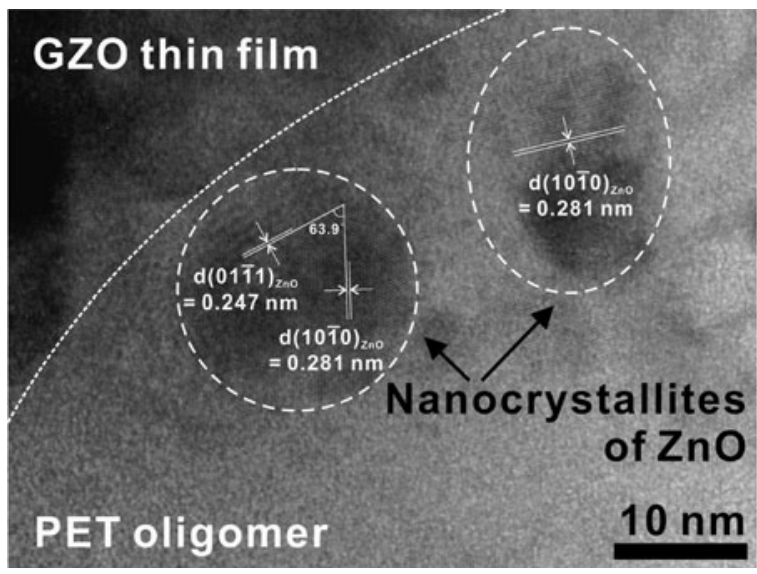

Figure 2. HRTEM micrograph of the $\mathrm{ZnO}$ nanocrystallites entrapped in PET oligomer. HRTEM, high-resolution transmission electron microscope; PET, polyethylene terephthalate.

the oligomer phases on both substrates. The GZO thin film on the PET oligomer has a narrow column width of $\sim 10 \mathrm{~nm}$. Furthermore, a small dark contrast was observed inside the oligomer phase, and it was found to be caused by $\mathrm{ZnO}$ nanocrystallites, as shown in Figure 2. However, the average column width of the GZO thin film on the PEN oligomer was around $20 \mathrm{~nm}$. Interestingly, the width of the column on the PEN oligomer phase was relatively narrow at the interface, and it became wider along with the growth direction.

The growth mechanisms of the GZO thin films on the oligomer phases are schematically drawn in Figure 3. At the initial growth stage, the nucleation of the GZO thin films occurs on the surface of the PET and the PEN substrates. The initially nucleated GZO nanocrystallites are spatially redistributed owing to the rapid growth of the oligomer phase in PET substrate, and they can be trapped inside the oligomer phase as shown in Figures 2 and 3a. The overgrown GZO thin film on the oligomer phase has a poor crystallinity owing to the loss of its initial nucleation sites. Eventually, the large extrusions of the GZO thin film grown on the oligomer phase and the nanocrystallites entrapped in the oligomer phase can be observed as shown in Figure 1a.

In the case of the PEN substrate, the GZO thin film was deposited on the oligomer phase without the critical loss of its crystallinity owing to the gradual growth of the oligomer, as depicted in Figure 3b. As the sputtering deposition of the GZO thin film proceeds, the initially nucleated columns grow to the normal direction to the surface of the oligomer. Consequently, the growth front of the column should become wider because of the radial distribution of the initially grown columns on the oligomer phase, as shown in Figures $1 \mathrm{~b}$ and $3 \mathrm{~b}$.

The effects of the type of plastic substrates on the thermal stability of the GZO thin film were also investigated. Figure 4 shows the results of the Hall measurements of the GZO thin films grown on the PET and the PEN substrates as a function of the thermal stability test time at $150^{\circ} \mathrm{C}$ in air. When $t=0$, the GZO thin film on PEN 
(a)
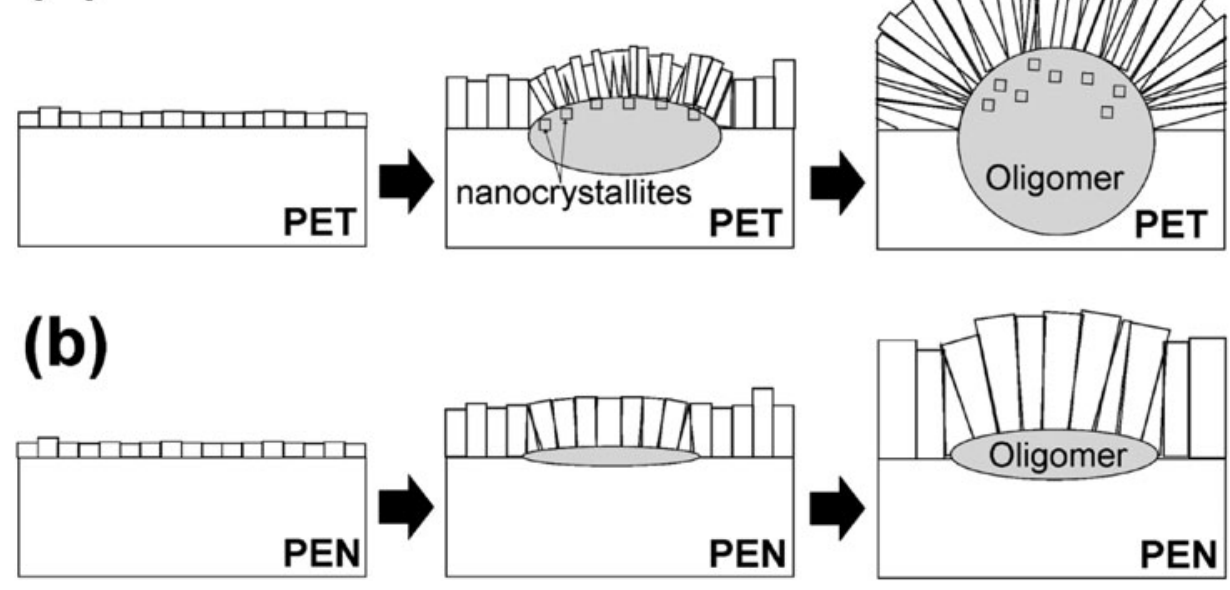

Figure 3. Schematic drawings for the growth mechanisms of GZO thin films on (a) PET oligomer and (b) PEN oligomer. GZO, Ga-doped ZnO; PET, polyethylene terephthalate; PEN, polyethylene naphthalate.

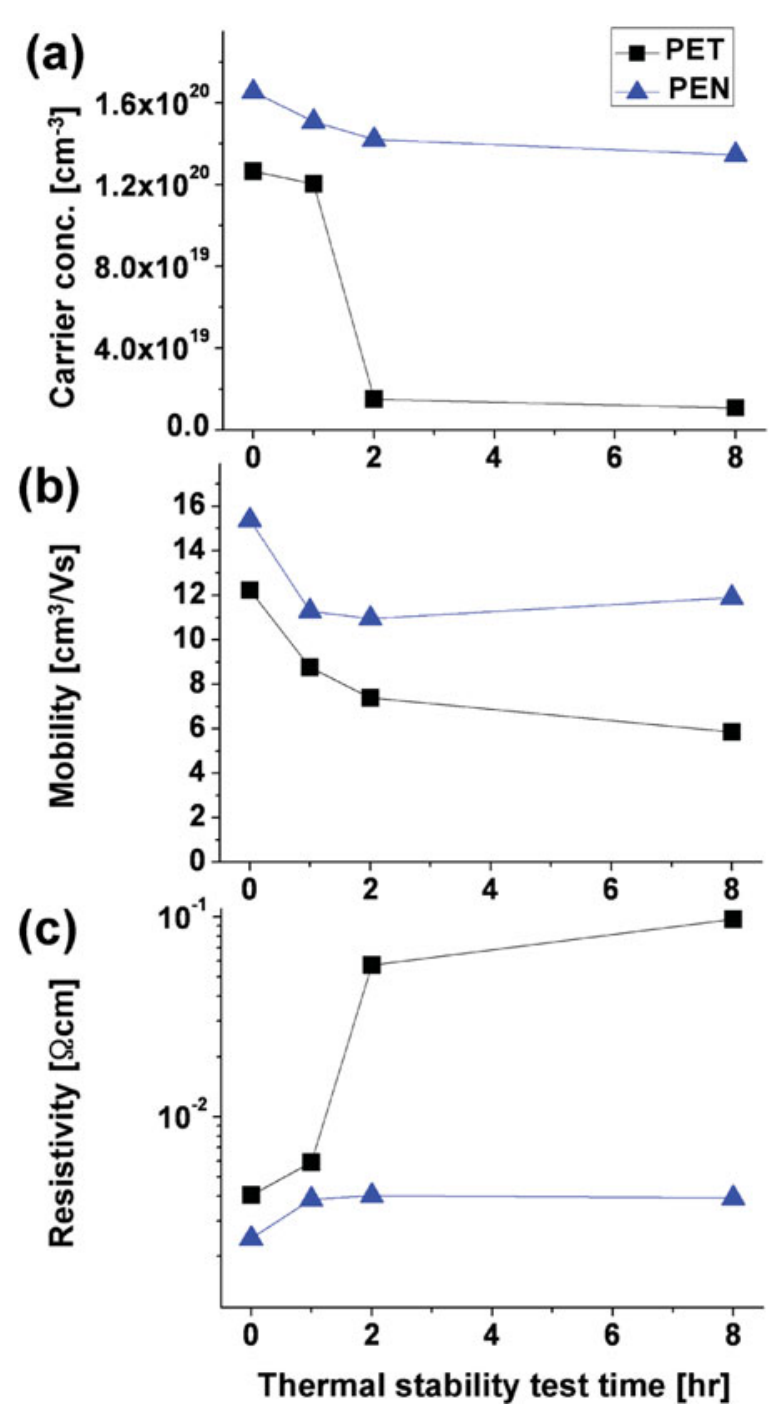

Figure 4. (a) Carrier concentration, (b) mobility, and (c) resistivity of Ga-doped $\mathrm{ZnO}$ thin films as a function of thermal stability test time at $150^{\circ} \mathrm{C}$ in air. substrate shows superior electrical properties compared with that on the PET substrate. As the thermal stability test time increased, both the carrier concentration and the Hall mobility of the GZO thin films decreased regardless of the type of the plastic substrates. Because the thermal stability tests were performed in air, oxygen, and water vapor molecules can be adsorbed at the grain boundary of the GZO thin film (Zhang \& Brodie, 1994; Sans et al., 2007; Bayraktaroglu et al., 2008). The adsorbed molecules can trap the free electrons in the GZO thin films, thus leading to a decrease in the carrier concentration (Zhang \& Brodie, 1994; Bayraktaroglu et al., 2008). Furthermore, the negatively charged molecules can act as a potential barrier to hinder the electronic transport by trapping the electrons (Zhang \& Brodie, 1994; Miyata et al., 2008). Therefore, a decrease in both the carrier concentration and the Hall mobility results in an increase in the resistivity, as shown in Figure 4c.

However, the tendency of the increase in resistivity was strongly dependent on the type of the plastic substrate. In the case of PEN substrate, the increase in the resistivity was observed after $1 \mathrm{~h}$, but it was saturated up to $8 \mathrm{~h}$. Meanwhile, there was a drastic increase in the resistivity after $2 \mathrm{~h}$ in the GZO thin film grown on PET substrate. Because the GZO thin films were grown on the PET and PEN substrates with the same thickness, the rapid increase of the resistivity on the PET substrate might be attributed to the defect formation due to interfacial stress under thermal condition rather than the degradation by the surface-adsorbed molecules.

Figure 5 shows optical micrographs of the as-deposited and the thermal stability-tested GZO thin films on the plastic substrates. Regardless of the substrate type, the asdeposited GZO thin films showed no crack, as shown in Figures $5 \mathrm{a}$ and $5 \mathrm{~d}$. After a 2-h test, the formation of the crack in the GZO thin film was observed on the PET substrate as marked by arrows. As the thermal stability time increased to $8 \mathrm{~h}$, the cracks on the PET substrate were more clearly observed. However, there was no crack in the GZO 


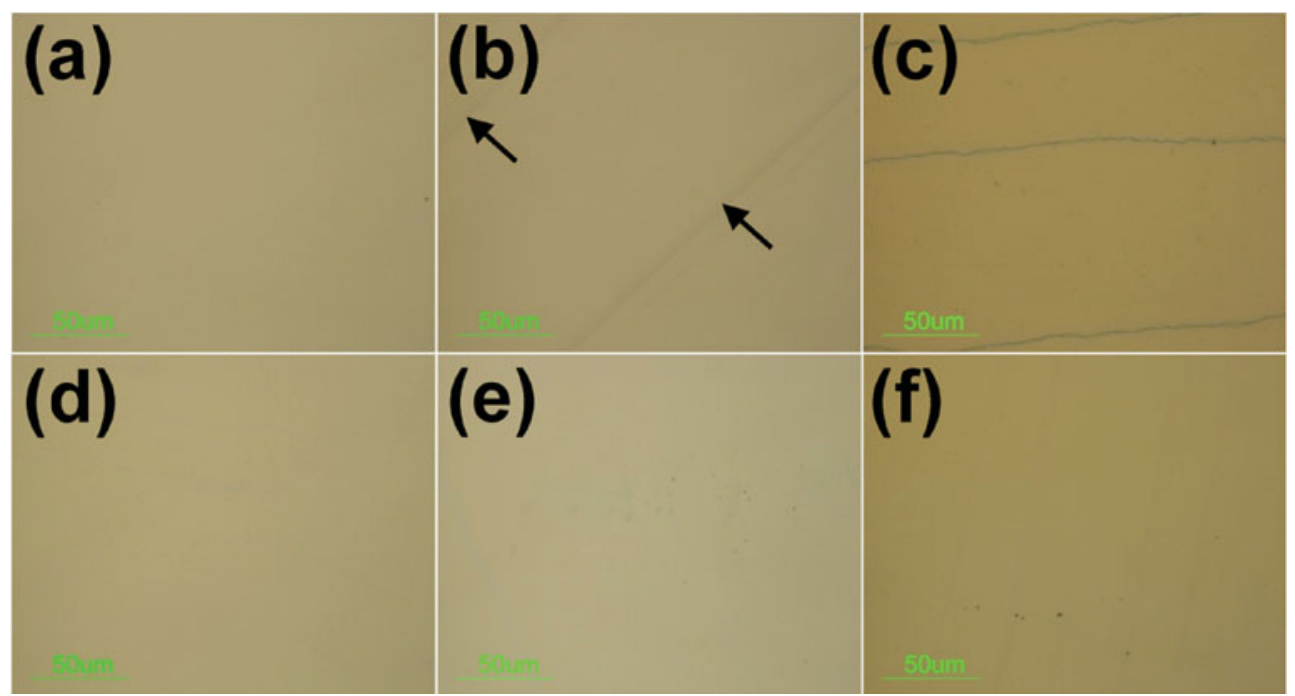

Figure 5. Optical micrographs of GZO thin films on PET substrate at (a) $t=0$, (b) $t=2 \mathrm{~h}$, and (c) $t=8 \mathrm{~h}$, and those on PEN substrates at (d) $t=0,(\mathbf{e}) t=2 \mathrm{~h}$, and (f) $t=8 \mathrm{~h}$. GZO, Ga-doped ZnO; PET, polyethylene terephthalate; PEN, polyethylene naphthalate

thin film on the PEN substrate up to $8 \mathrm{~h}$. The behavior of the crack formation was strongly correlated with the electrical properties of the GZO thin films. As shown in Figure 4, the GZO thin film on the PET substrate indicated poor thermal stability compared with that on the PEN substrate. In particular, an abrupt increase of the resistivity was observed in the GZO thin film on the PET substrate after the 2-h test, and it could have originated from the crack formation, as shown in Figure 5b.

The difference in behavior of the crack formation is dependent on the CTE of the plastic substrate. In general, the CTE of plastic substrates is around one order higher than that of $\mathrm{ZnO}\left(2.9-4.75 \times 10^{-6} /{ }^{\circ} \mathrm{C}\right.$; MacDonald et al., 2005; Morkoç \& Özgür, 2009). Because the CTE of the PET substrate $\left(15 \times 10^{-6} /{ }^{\circ} \mathrm{C}\right)$ is $\sim 15 \%$ larger than that of the PEN substrate $\left(13 \times 10^{-6} /{ }^{\circ} \mathrm{C}\right)$, thermal stress can be more strongly exerted on the GZO/PET interface than on the GZO/PEN interface during the thermal stability test at $150^{\circ} \mathrm{C}$ (MacDonald et al., 2005). Furthermore, the sharp extrusions of oligomer phase on the PET substrate can provide the easy sites for the nucleation of the crack, because the stress can be concentrated at the points. Therefore, it can be concluded that the PEN substrate is more suitable than PET substrate for the deposition of the GZO thin film from the view point of the interfacial structure and the thermal stability.

\section{SUMMARY}

The interfacial structure and the thermal stability of GZO thin films on plastic substrates were investigated. PET and PEN substrates were used for the sputtering deposition of the GZO thin films. During the deposition, oligomer phases were produced at the interface between the GZO thin films and the plastic substrate owing to the energetic bombardment of sputtered species. The size of the oligomer phase on the PET substrate was much bigger than that on the PEN substrate, and it was strongly affected by the molecular structure of the polymer chain. The microstructure of the GZO thin films grown on the oligomer phases were characterized by using TEM, and the growth mechanisms on the PET and the PEN substrate were, respectively, proposed. Thermal stability tests of the GZO thin films on the plastic substrates were carried out at $150^{\circ} \mathrm{C}$ for up to $8 \mathrm{~h}$ in air. An abrupt increase in the resistivity in the GZO thin film on the PET substrate was observed after $2 \mathrm{~h}$, and it was related to the crack formation by the thermal stress originating from the high CTE difference between the thin film and the substrate. In the case of PEN substrate, a drastic increase of the resistivity and crack formation in the GZO thin film was not observed up to $8 \mathrm{~h}$.

\section{ACKNOWLEDGMENT}

This work was supported by a Grant-in-Aid for R\&D Programs (No. 10029940) from the Korean Ministry of Knowledge Economy.

\section{RefERENCES}

Bayraktaroglu, B., Leedy, K. \& Bedford, R. (2008). High temperature stability of postgrowth annealed transparent and conductive ZnO:Al films. Appl Phys Lett 93, 022104.

Bishop, C.A. (2010). Roll-to-Roll Vacuum Deposition of Barrier Coatings. Leicestershire, UK: Wiley.

Cheng, C. \& Wagner, S. (2009). Overview of flexible electronics technology. In Flexible Electronics: Materials and Applications, Wong, W.S. \& Salleo, A. (Eds.), pp. 1-28. New York: Springer.

Ellmer, K. \& Mientus, R. (2008). Carrier transport in polycrystalline transparent conductive oxides: A comparative study of zinc oxide and indium oxide. Thin Solid Films 516, 4620-4627.

Fortunato, E., Nunes, P., Marques, A., Costa, D., Águas, H., Ferreira, I., Costa, M.E.V., Godinho, M.H., Almeida, P.L., Borges, J.P. \& MArtins, R. (2002). Transparent, conductive 
$\mathrm{ZnO}$ :Al thin film deposited on polymer substrates by RF magnetron sputtering. Surf Coat Tech 151/152, 247-251.

Kim, D.W., Kang, J.-H., Lim, Y.S., Lee, M.-H., Seo, W.-S., Park, H.-H., SEO, K.H. \& PARK, M.G. (2010). Effect of a hard coating layer on the damp heat stability of Ga-doped $\mathrm{ZnO}$ thin films on polyethylene terephthalate substrates. J Kor Phys Soc 57, 1045-1048.

Kim, D.W., Kang, J.-H., Lim, Y.S., Lee, M.-H., Seo, W.-S., ParK, H.-H., Seo, K.H. \& PARK, M.G. (2011). Pretreatment of polyethylene terephthalate substrate for the growth of Ga-doped ZnO thin film. J Nanosci Nanotech 11, 1617-1620.

Koidis, C., Logothetidis, S., Laskarakis, A., Tsiaoussis, I. \& Frangis, N. (2009). Thin film and interface properties during $\mathrm{ZnO}$ deposition onto high-barrier hybrid/PET flexible substrates. Micron 40, 130-134.

MacDonald, B.A., Rollins, K., MacKerron, D., Rakos, K., Eveson, R., Наsнimoto, K. \& Rustin, B. (2005). Engineered films for display technologies. In Flexible Flat Panel Displays, MacDonald, B.A. (Ed.), pp. 11-33. Chichester, UK: Wiley.

Minami, T. (2000). New n-type transparent conducting oxides. Mater Res Soc Bull 25, 38-44.
Miyata, T., Ohtani, Y., Kuboi, T. \& Minami, T. (2008). Stability of nano-thick transparent conducting oxide films for use in a moist environment. Thin Solid Films 516, 1354-1358.

Morkoç, H. \& Özgür, Ü. (2009). Zinc Oxide: Fundamentals, Materials and Device Technology. Weinheim, Germany: Wiley-VCH.

Reichlmaier, S., Bryan, S.R. \& Briggs, D. (1995). Surface trimer crystallization on poly (ethylene terephthalate) studied by timeof-flight secondary ion mass spectrometry. J Vac Sci Technol A 13, 1217-1223.

Sans, A., Martinez-Criado, G., Pellicer-Porres, J., SanchezRoyo, J.F. \& Segura, A. (2007). Thermal instability of electrically active centers in heavily Ga-doped $\mathrm{ZnO}$ thin films: X-ray absorption study of the Ga-site configuration. Appl Phys Lett 91, 221904.

Yasuda, H. (1978). Glow discharge polymerization. In Thin Film Processes, Vossen, J.L. \& Kern, W. (Eds.), pp. 361-398. New York: Academic Press.

Zhang, D.H. \& Brodie, D.E. (1994). Effects of annealing ZnO films prepared by ion-beam-assisted reactive deposition. Thin Solid Films 238, 95-100. 